Pacific

Journal of

Mathematics

ON THE EXISTENCE

OF LARGE DEGREE GALOIS REPRESENTATIONS FOR FIELDS OF SMALL DISCRIMINANT

JEREMY ROUSE AND FRANK THORNE 


\title{
ON THE EXISTENCE \\ OF LARGE DEGREE GALOIS REPRESENTATIONS FOR FIELDS OF SMALL DISCRIMINANT
}

\author{
JEREMY ROUSE AND FRANK THORNE
}

\begin{abstract}
Let $L / K$ be a Galois extension of number fields. We prove two lower bounds on the maximum of the degrees of the irreducible complex representations of $\operatorname{Gal}(L / K)$, the sharper of which is conditional on the Artin conjecture and the generalized Riemann hypothesis. Our bound is nontrivial when [ $K$ : $\mathbb{Q}]$ is small and $L$ has small root discriminant, and might be summarized as saying that such fields can't be "too abelian".
\end{abstract}

\section{Introduction}

It is known that the discriminant of a number field cannot be too small. Minkowski's work on the geometry of numbers implies that

$$
|\operatorname{Disc}(K)|>\left(\frac{e^{2} \pi}{4}-o(1)\right)^{[K: \mathbb{Q}]} ;
$$

we write this bound as $\operatorname{rd}_{K}>e^{2} \pi / 4-o(1)$, where $\operatorname{rd}_{K}:=(|\operatorname{Disc}(K)|)^{1 /[K: \mathbb{Q}]}$ is the root discriminant of $K$. These bounds can be improved by using analytic properties of the Dedekind zeta function of $K$, and this was noticed by Stark (see the parenthetical comment in the proof of Lemma 4 on page 140 of [Stark 1974]), and worked out in detail by Andrew Odlyzko [1976] in his MIT dissertation (supervised by Stark). The sharpest known bounds, due to Poitou [1977] (see also [Odlyzko 1990]), are

$$
\operatorname{rd}_{K} \geq(60.8395 \ldots)^{r_{1} /[K: \mathbb{Q}]}(22.3816 \ldots)^{2 r_{2} /[K: \mathbb{Q}]}-O\left([K: \mathbb{Q}]^{-2 / 3}\right),
$$

where $[K: \mathbb{Q}]=r_{1}+2 r_{2}$, and $r_{1}$ and $r_{2}$ are the numbers of real and complex embeddings of $K$, respectively. (The error term in (1-1) can be improved.) If one assumes the generalized Riemann hypothesis, the constants above can be improved to $215.3325 \ldots$ and $44.7632 \ldots$ respectively.

Conversely, Golod and Shafarevich [1964] proved that these bounds are sharp apart from the constants, by establishing the existence of infinite class field towers

MSC2010: primary 11R29; secondary 11R42.

Keywords: Artin $L$-function, Galois representation, Rankin-Selberg $L$-function. 
$K_{1} \subseteq K_{2} \subseteq K_{3} \subseteq \cdots$ where each $K_{i+1} / K_{i}$ is abelian and unramified, so that each field $K_{i}$ has the same root discriminant. Martinet [1979] gave the example $K_{1}=\mathbb{Q}\left(\zeta_{11}+\zeta_{11}^{-1}, \sqrt{-46}\right)$, which has an infinite 2-class field tower of root discriminant $92.2 \ldots$, and Hajir and Maire [2001; 2002] constructed a tower of fields with root discriminants bounded by 82.2 , in which tame ramification is allowed.

It is expected that fields of small discriminant should be uncommon. For example, Odlyzko [1990] asked whether there are infinitely many fields of prime degree of bounded root discriminant; such fields cannot be constructed via class field towers. Several researchers have studied this question in small degree. Jones and Roberts [2007] studied the set of Galois number fields $K / \mathbb{Q}$ with certain fixed Galois groups $G$; for a variety of groups, including $A_{4}, A_{5}, A_{6}, S_{4}, S_{5}, S_{6}$, they proved that $\operatorname{rd}_{K}>44.7632 \ldots$ apart from a finite list of fields $K$ which they compute explicitly. Voight [2008] studied the set of all totally real number fields $K$ with $\operatorname{rd}_{K} \leq 14$, finding that there are exactly 1229 such fields, each with $[K: \mathbb{Q}] \leq 9$.

In light of this work, it is natural to ask whether Galois extensions of small absolute discriminant must have any special algebraic properties. (The analogous problems for nonnormal extensions are much more delicate.) The easiest result to prove is that they cannot be abelian, and we carry this out over $\mathbb{Q}$ in the introduction (starting with (1-4)). In [Leshin 2013], it is proven that, given a number field $K$, a positive integer $n$, and real number $N$, there are only finitely many Galois extensions $L / K$ with $\operatorname{Gal}(L / K)$ solvable with derived length $\leq n$ and with $\operatorname{rd}_{L} \leq N$. In this paper, we study the representation theory of Galois groups of extensions of small discriminant, and prove that such Galois groups must have (relatively) large degree complex representations.

We will prove two versions of this result. The first is the following:

Theorem 1.1. Let $L / K$ be a Galois extension and let $r$ be the maximum of the degrees of the irreducible complex representations of $\mathrm{Gal}(L / K)$. Then there is a constant $C_{1}$ so that

$$
r \geq \frac{1}{\log \operatorname{rd}_{L}}\left(C_{1} \frac{\log \log [L: \mathbb{Q}]}{\log \log \log [L: \mathbb{Q}]}-\log [K: \mathbb{Q}]\right) .
$$

Remark. The bound of course only makes sense for large $[L: \mathbb{Q}]$. A straightforward but somewhat lengthy calculation shows that we may take $C_{1}=\frac{1}{16}$ provided $[L: \mathbb{Q}] \geq e^{e^{8}}$.

The basic idea of the proof is to regard $L$ as an abelian extension of an intermediate field $F$ of small degree. The existence of such an $F$ follows from Theorem 12.23 of [Isaacs 2006], which states that if $G$ is a finite group with the property that all of its irreducible representations have degree $\leq r$, then $G$ must have an abelian subgroup of index $\leq(r !)^{2}$. (There is also a converse given in Problem 2.9 of [Isaacs 2006]: if $G$ has an irreducible representation of degree $>r$, then $G$ cannot have an abelian 
subgroup of index $\leq r$.) We may then adapt our proof for the abelian case to prove that either $L$ has small root discriminant, or $F$ has relatively large degree.

It is also possible to study the representations of $\operatorname{Gal}(L / \mathbb{Q})$ directly, without first passing to an intermediate extension $F$, via Artin $L$-functions. We were unable to improve upon Theorem 1.1 this way, but under the hypothesis that Artin $L$-functions are well behaved we prove the following improvement of Theorem 1.1:

Theorem 1.2. Assume that all Artin L-functions are entire and satisfy the Riemann hypothesis. There is a positive constant $C_{2}$ so that if $L / K$ is any Galois extension of number fields of degree $d$, then $\operatorname{Gal}(L / K)$ must have an irreducible complex representation of degree at least

$$
\frac{C_{2}(\log [L: \mathbb{Q}])^{1 / 5}}{\left(\log \operatorname{rd}_{L}\right)^{2 / 5}[K: \mathbb{Q}]^{3 / 5}}
$$

Remark. Two issues arise when attempting to prove an unconditional version of this result. The first is that the unconditional zero-free regions for $L$-functions have implied constants that depend quite badly on the degree of the $L$-function involved. (See for example Theorem 5.33 of [Iwaniec and Kowalski 2004].) The second is the presence of the possible exceptional zero. Without accounting for the exceptional zero issue, it seems that the best lower bound we can obtain using the zero-free regions mentioned above is $r \gg \sqrt{\log \log [L: \mathbb{Q}]}$, for an implied constant depending on $K$ and on $\operatorname{rd}_{L}$.

We now illustrate the nature of our question by handling the case where $L / \mathbb{Q}$ is abelian of degree $>2$. By Kronecker-Weber we have $L \subseteq \mathbb{Q}\left(\zeta_{n}\right)$ for some $n$, and

$$
\zeta_{L / \mathbb{Q}}(s)=\zeta(s) \prod_{i=2}^{[L: \mathbb{Q}]} L\left(s, \chi_{i}\right),
$$

where $\chi_{i}$ are Dirichlet characters of conductor $N_{i}$ for some $N_{i} \mid n$. We have $\operatorname{Disc}(L)=\prod_{i} N_{i}$, and therefore

$$
\log \operatorname{rd}_{L}=\frac{1}{[L: \mathbb{Q}]} \sum_{i=2}^{[L: \mathbb{Q}]} \log N_{i} .
$$

Let $M:=\sqrt{[L: \mathbb{Q}] / 2}$. There are at most $M^{2}$ Dirichlet characters with conductor $\leq M$, so that with $[L: \mathbb{Q}]=2 M^{2}$ the right side of (1-5) is greater than $\frac{1}{2} \log M$, so that

$$
\operatorname{rd}_{L}>\exp \left(\frac{1}{4} \log \left(\frac{1}{2}[L: \mathbb{Q}]\right)\right),
$$

a bound of the same shape as our theorems. Although this proof is not complicated, it makes essential use of class field theory and it seems that the use of sophisticated 
tools cannot be avoided. We could improve our bound somewhat, but note that it is already stronger than the (conditional) bound $\log \operatorname{rd}_{L} \geq C_{2}(\log [L: \mathbb{Q}])^{1 / 5}$ implied by Theorem 1.2. Observe also that for $L=\mathbb{Q}\left(\zeta_{p}\right)$ we have $\operatorname{rd}_{L}=p^{(p-2) /(p-1)}$ and $[L: \mathbb{Q}]=p-1 \approx \operatorname{rd}_{L}$, implying a limit on the scope for improvement.

As an application of Theorem 1.2 we can say something about unramified extensions of a fixed number field $K$. Of course, the maximal unramified abelian extension of $K$ is the Hilbert class field of $K$ and the degree of this extension is $h_{K}$, the order of the ideal class group. However, there are number fields $K$ with Galois extensions $L$ unramified at all finite primes so that $\operatorname{Gal}(L / K)$ has no nontrivial abelian quotients. One of Artin's favorite examples is $K=\mathbb{Q}(\sqrt{2869})$, where if $L$ is the splitting field of $x^{5}-x-1$ over $\mathbb{Q}$, then $L / K$ is unramified and $\operatorname{Gal}(L / K) \cong A_{5}$.

Corollary 1.3. Assume that all Artin L-functions are entire and satisfy the Riemann hypothesis. Let $L_{1} / K, L_{2} / K, \ldots, L_{N} / K$ be linearly disjoint unramified Galois extensions and suppose that $\mathrm{Gal}\left(L_{i} / K\right)$ has an irreducible representation of degree $r$ for $1 \leq i \leq N$. Then there is a constant $C_{3}$ so that

$$
\log N \leq C_{3} r^{5} \log ^{2}(|\operatorname{Disc}(K)|)[K: \mathbb{Q}] .
$$

Remark. The main theorem proven in [Ellenberg and Venkatesh 2006] shows that the number $M$ of degree $n$ unramified extensions of $K$ satisfies

$$
\log M \ll_{\epsilon} n^{\epsilon}\left(n \log |\operatorname{Disc}(K)|+C_{4}[K: \mathbb{Q}]\right)
$$

for a constant $C_{4}$ depending on $n$. Because the power of $\log |\operatorname{Disc}(K)|$ is smaller, this result is better for fixed $n$ and varying $K$. However, since the size of $C_{4}$ is not specified, our result is better for fixed $K$ and varying $n$.

Remark. Another potential application occurs in the case when $r=2$ and $K=\mathbb{Q}$. Our theorem gives bounds on the number of degree-2 Artin $L$-functions with conductor bounded by $q$. In the odd case, these arise from weight- 1 newforms of level $q$, and in the even case, these arise (conjecturally) from Maass forms with eigenvalue $\frac{1}{4}$. However, we obtain bounds that are worse than polynomial in $q$. Michel and Venkatesh [2002] used the Petersson-Kuznetsov formula to obtain bounds of the form $q^{c+\epsilon}$, where $c$ is a constant depending on the type of representation (dihedral, tetrahedral, octahedral, or icosahedral).

Throughout the paper we use the notation $|\operatorname{Disc}(K)|$ for the absolute value of the discriminant of $K, \mathcal{O}_{K}$ for the ring of integers of $K, \operatorname{rd}_{K}=|\operatorname{Disc}(K)|^{1 /[K: \mathbb{Q}]}$, $\mathcal{N}_{K / \mathbb{Q}}(\mathfrak{a})$ for the norm from $K$ to $\mathbb{Q}$ of an ideal of $\mathbb{O}_{K}, h_{K}$ for the order of the ideal class group of $\mathfrak{O}_{K}$, and $\mathfrak{d}_{L / K}$ for the relative discriminant of $L$ over $K$. We denote by $C_{1}, C_{2}, \ldots$ a sequence of absolute constants. We also occasionally write $f \ll g$ to mean $f \leq C g$ for some constant $C$, absolute unless otherwise noted. 
We provide a little bit of preliminary background in Section 2, and then we prove Theorem 1.1 in Section 3 and Theorem 1.2 and Corollary 1.3 in Section 4.

\section{Background on number fields, discriminants, and conductors}

In this section we briefly recall a few facts related to zeta and $L$-functions associated to number fields, used in the proofs of both Theorem 1.1 and Theorem 1.2.

The Dedekind zeta function of a number field $L$ is given by the Dirichlet series

$$
\zeta_{L}(s)=\sum \mathcal{N}_{L / \mathbb{Q}}(\mathfrak{a})^{-s},
$$

where the sum is over integral ideals of $O_{L}$. For a Galois extension $L / K$, this zeta function enjoys the factorization

$$
\zeta_{L}(s)=\zeta_{L / K}(s)=\prod_{\rho \in \operatorname{Irr}(\operatorname{Gal}(L / K))} L(s, \rho)^{\operatorname{deg} \rho},
$$

where $\rho$ varies over all irreducible complex representations of $G:=\operatorname{Gal}(L / K)$, and $L(s, \rho)$ is the associated Artin $L$-function. (For background on Artin $L$-functions see [Neukirch 1999]; see p. 524 for the proof of (2-2) in particular.)

This formula is the nonabelian generalization of (1-4). In general, it is not known that the $L(s, \rho)$ are "proper" $L$-functions (as defined on [Iwaniec and Kowalski 2004, p. 94] for example) and in particular that they are holomorphic in the critical strip. However, this was conjectured by Artin; we refer to this assumption as the Artin conjecture and assume its truth in Section 4.

Remark. As a consequence of Brauer's theorem on group characters [Neukirch 1999, p. 522], it is known that the Artin $L$-functions are quotients of Hecke $L$ functions, and therefore meromorphic, and this suffices in many applications. For example, Lagarias and Odlyzko [1977] used this fact to prove an unconditional and effective version of the Chebotarev density theorem.

If $\operatorname{Gal}(L / K)$ is abelian, then the representations are all one-dimensional, and class field theory establishes that the characters of $\mathrm{Gal}(L / K)$ coincide with Hecke characters of $L / K$, so that (2-2) becomes

$$
\zeta_{L / K}(s)=\zeta_{K}(s) \prod_{i=2}^{[L: K]} L\left(s, \chi_{i}\right),
$$

where the product ranges over Hecke characters of $K$. As in our application of (1-4), we will argue that there cannot be too many characters $\chi$ or representations $\rho$ of small conductor (and, in the latter case, of bounded degree). 
We can use (2-2) to derive more general versions of (1-5): it follows [Neukirch 1999, p. 527] from (2-2) that the relative discriminant $\mathfrak{d}(L / K)$ satisfies the formula

$$
\mathfrak{d}(L / K)=\prod_{\rho \in \operatorname{Irr}(G)} \mathfrak{f}(\rho)^{\operatorname{deg} \rho},
$$

where the ideal $\mathfrak{f}(\rho)$ of $K$ is the Artin conductor associated to $\rho$.

If $L / K$ is abelian then we can write this as $\mathfrak{d}(L / K)=\prod_{\chi_{i}} \mathfrak{f}\left(\chi_{i}\right)$. Taking norms down to $\mathbb{Q}$ and using the relation [Neukirch 1999, p. 202]

$$
|\operatorname{Disc}(L)|=|\operatorname{Disc}(K)|^{[L: K]} \mathcal{N}_{K / \mathbb{Q}}\left(\mathfrak{d}_{L / K}\right),
$$

we obtain

$$
\log \operatorname{rd}_{L}=\log \operatorname{rd}_{K}+\frac{1}{[L: \mathbb{Q}]} \sum_{i} \log \mathcal{N}_{K / \mathbb{Q}} \mathfrak{f}\left(\chi_{i}\right)
$$

If $L / K$ is not necessarily abelian, then the conductor $q(\rho)$ of $L(s, \rho)$ is related to $\mathfrak{f}(\rho)$ by the formula

$$
q(\rho)=|\operatorname{Disc}(K)|^{\operatorname{deg} \rho} \mathcal{N}_{K / \mathbb{Q}} \mathfrak{f}(\rho) .
$$

Taking absolute norms in (2-4), multiplying by $|\operatorname{Disc}(K)|^{d}$, and again using (2-5) we obtain

$$
|\operatorname{Disc}(L)|=\prod_{\rho \in \operatorname{Irr}(G)} q(\rho)^{\operatorname{deg} \rho} .
$$

\section{Proof of Theorem 1.1}

We first prove a lemma bounding some quantities which occur in the proof.

Lemma 3.1. For a number field $F$ of degree $f$, the following hold:

(1) The number of ideals $\mathfrak{a}$ of $\mathcal{O}_{F}$ with $\mathcal{N}(\mathfrak{a})<Y$ is bounded by eY $(1+\log Y)^{f}$.

(2) We have $h_{F}<e|\operatorname{Disc}(F)|^{1 / 2}\left(1+\frac{1}{2} \log |\operatorname{Disc}(F)|\right)^{f}$.

Proof. This is standard and we give an easy proof inspired by [Cojocaru and Murty 2006, p. 68]. We have that $\zeta_{F}(s)=\sum_{n=1}^{\infty} a_{n}(F) / n^{s}$, where $a_{n}(F)$ is the number of integral ideals of norm $n$ in $\mathscr{O}_{F}$. The coefficientwise bound $\zeta_{F}(s)<\zeta(s)^{f}=$ $\sum_{n} d_{f}(n) n^{-s}$ yields that, for $\sigma>1$,

$$
\sum_{n<Y} d_{f}(n)<\sum_{n} d_{f}(n)(Y / n)^{\sigma}=Y^{\sigma} \zeta(\sigma)^{f} .
$$

We now choose $\sigma=1+1 / \log Y$, and use the fact that $\zeta(\sigma)<1+\frac{1}{\sigma-1}$ for $\sigma>1$. The second part follows from the classical Minkowski bound (see for example [Neukirch 1999, Chapter 1.6]), which implies that each ideal class in $O_{F}$ is represented by an ideal $\mathfrak{a}$ with $\mathcal{N}(\mathfrak{a})<\sqrt{|\operatorname{Disc}(F)|}$. 
Proof of Theorem 1.1. The proof is similar to that of (1-6), but we will need to work with messier inequalities.

By the character theory remarks after the theorem, $L$ has a subfield $F$ for which $L / F$ is abelian, such that $[F: K] \leq(r !)^{2}<r^{2 r}$. We assume that $[L: \mathbb{Q}]$ and therefore $\operatorname{rd}_{L}$ are bounded below by absolute constants $\left([L: \mathbb{Q}] \geq e^{e^{8}}\right.$ suffices). Depending on the relative sizes of these quantities, we will see that either

$$
r \geq C_{5} \frac{\log \log [L: \mathbb{Q}]}{\log \log \log [L: \mathbb{Q}]}-\log [K: \mathbb{Q}]
$$

or

$$
\log \operatorname{rd}_{L} \geq C_{6} \log \log [L: \mathbb{Q}]
$$

for positive constants $C_{5}$ and $C_{6}$, implying the theorem. There is no obstacle to determining particular values for these constants, but for simplicity we omit the details.

We begin with the generalization (2-6) of (1-5), which said that

$$
\log \operatorname{rd}_{L}=\log \operatorname{rd}_{F}+\frac{1}{[L: \mathbb{Q}]} \sum_{i} \log \mathcal{N}_{F / \mathbb{Q}} \mathfrak{f}\left(\chi_{i}\right),
$$

where $\chi_{i}$ are distinct Hecke characters of $F$. The number of characters of conductor $\mathfrak{m}$ is less than $2^{[F: \mathbb{Q}]} h_{F} \mathcal{N}(\mathfrak{m})$ [Milne 2011, Theorem V.1.7, p. 146], and Lemma 3.1 bounds both $h_{F}$ and the number of $\mathfrak{m}$ which can appear, so that for $Y \geq 1$ the number of characters whose conductor has norm $\leq Y$ is bounded above by $e^{2} Y^{2}|\operatorname{Disc}(F)|^{1 / 2}\left(2+\log \left(Y^{2}|\operatorname{Disc}(F)|\right)\right)^{2[F: \mathbb{Q}]}$.

Given $[L: \mathbb{Q}]$ and $[F: \mathbb{Q}]$, suppose that $Y>e^{-1}|\operatorname{Disc}(F)|^{-1 / 2}$ is defined by the equation

$$
\frac{[L: \mathbb{Q}]}{2[F: \mathbb{Q}]}=e^{2} Y^{2}|\operatorname{Disc}(F)|^{1 / 2}\left(2+\log \left(Y^{2}|\operatorname{Disc}(F)|\right)\right)^{2[F: \mathbb{Q}]},
$$

so that in (3-3) there are at least $\frac{[L: \mathbb{Q}]}{2[F: \mathbb{Q}]}$ characters of conductor $>Y$, and hence

$$
\log \operatorname{rd}_{L} \geq \log \operatorname{rd}_{F}+\frac{1}{2[F: \mathbb{Q}]} \log Y .
$$

(Observe that we do not necessarily have $Y>1$, for example if $L$ is the Hilbert class field of $F$.) We divide our analysis of (3-5) into three cases and prove that each implies (3-1) or (3-2).

Large discriminant. If $\operatorname{Disc}(F) \geq[L: \mathbb{Q}]^{1 / 10}$, we ignore (3-5) and instead note that $\log \operatorname{rd}_{F} \geq \log [L: \mathbb{Q}] /(10[F: \mathbb{Q}])$, and so

$$
\log \operatorname{rd}_{L} \geq \frac{1}{10 r^{2 r}[K: \mathbb{Q}]} \log [L: \mathbb{Q}],
$$


and we obtain at least one of (3-1) and (3-2) depending on whether $r^{2 r}[K: \mathbb{Q}]>$ $(\log [L: \mathbb{Q}])^{1 / 2}$ or not.

We assume henceforth that $\operatorname{Disc}(F)<[L: \mathbb{Q}]^{1 / 10}$, which implies that $[F: \mathbb{Q}]<$ $\frac{1}{10} \log [L: \mathbb{Q}]$ for $[F: \mathbb{Q}] \geq 3$, and write $Y^{\prime}:=\max (Y, 100)$ and $Z:=Y^{\prime 2}|\operatorname{Disc}(F)|^{1 / 2}$.

Small discriminant and large degree. Assume to start with that either $Z \leq(2+$ $2 \log Z)^{2[F: \mathbb{Q}]}$ or $Y<100$. Applying our upper bounds on $\operatorname{Disc}(F),[F: \mathbb{Q}]$, and $Z$, we see that

$$
[L: \mathbb{Q}]^{4 / 5} \leq C_{7}\left(4 \log \left(Y^{\prime 2}|\operatorname{Disc}(F)|\right)\right)^{4[F: \mathbb{Q}]} .
$$

Taking logarithms and applying the bound ${ }^{1} Y^{\prime 2}|\operatorname{Disc}(F)|<[L: \mathbb{Q}]$, we obtain

$$
\log [L: \mathbb{Q}] \leq C_{8}[F: \mathbb{Q}] \log \log [L: \mathbb{Q}],
$$

so that $\log [L: \mathbb{Q}] / \log \log [L: \mathbb{Q}] \leq C_{9}[K: \mathbb{Q}] r^{2 r}$, which implies that

$$
r \geq C_{10} \frac{\log \log [L: \mathbb{Q}]}{\log \log \log [L: \mathbb{Q}]}-\log [K: \mathbb{Q}] .
$$

Small discriminant and small degree. Finally, assume that $Z>(2+2 \log Z)^{2[F: \mathbb{Q}]}$ and $Y \geq 100$. Then $[F: \mathbb{Q}] \leq C_{11} \log Z / \log \log Z$, and our bound on $\operatorname{Disc}(F)$ implies that $\log Z \leq C_{12} \log Y$, so that $[F: \mathbb{Q}] \leq C_{13} \log Y / \log \log Y$. We thus have $\log Y /(2[F: \mathbb{Q}]) \geq C_{14} \log \log Y$, and so, by (3-5),

$$
\log \operatorname{rd}_{L} \geq \log \operatorname{rd}_{F}+\frac{1}{2[F: \mathbb{Q}]} \log Y \geq \log \operatorname{rd}_{F}+C_{14} \log \log Y .
$$

Finally, (3-4) implies that $\log Y \leq \frac{1}{2} \log [L: \mathbb{Q}]$, giving us

$$
\log \operatorname{rd}_{L} \geq \log \operatorname{rd}_{F}+C_{15} \log \log [L: \mathbb{Q}] .
$$

This completes our list of cases, and hence the proof.

\section{Proof of Theorem 1.2}

In the proof we will assume familiarity with Artin $L$-functions and Rankin-Selberg convolutions, as described in [Neukirch 1999] (and Section 2) and [Iwaniec and Kowalski 2004], respectively. We also assume the truth of the Artin conjecture. There is no theoretical obstacle to carrying out the methods of this section without any unproved hypotheses, but when we tried this the error terms in (4-6) were too large to be of interest.

As in the nonabelian case, we need to bound the number of possible $q(\rho)$ of bounded conductor (and now also of bounded degree). However, in general the representations $\rho$ are not (yet!) known to correspond to arithmetic objects which

\footnotetext{
${ }^{1}$ If $Y^{\prime}=100$, this follows from $\operatorname{Disc}(F)<[L: \mathbb{Q}]^{1 / 10}$. If $Y^{\prime}=Y$, this follows from (3-4).
} 
might be more easily counted. Instead, in Proposition 4.1 we (conditionally) bound the number of possible $L$-functions. Assuming GRH and the Artin conjecture, we will see that any two such Artin $L$-functions must have rather different Dirichlet series representations, because their Rankin-Selberg convolution cannot have a pole. A pigeonhole-type argument will then allow us to bound the number of possible $L$-functions.

After proving Proposition 4.1 we will conclude as before. In brief, if $L / K$ is a Galois extension of large degree with many representations of small degree, then many of these representations will have large conductor, and so $L$ will have large discriminant.

Proposition 4.1. Assume that the Artin conjecture and Riemann hypothesis hold for Artin L-functions, and let $\rho$ and $\rho^{\prime}$ be distinct irreducible nontrivial representations of $\mathrm{Gal}(L / K)$ of degree $r$ and conductor $\leq q$ (as defined in (2-7)). The Artin L-series of $\rho$ and $\rho^{\prime}$ have Euler products

$$
L(s, \rho)=\prod_{\mathfrak{p}} \prod_{i=1}^{d}\left(1-\alpha_{i, \rho}(\mathfrak{p}) N(\mathfrak{p})^{-s}\right)^{-1}, \quad L\left(s, \rho^{\prime}\right)=\prod_{\mathfrak{p}} \prod_{i=1}^{d}\left(1-\alpha_{i, \rho^{\prime}}(\mathfrak{p}) N(\mathfrak{p})^{-s}\right)^{-1} .
$$

Assume that $\log q>r[K: \mathbb{Q}]$. Then, for $X \geq C_{16} r^{2} \log ^{2} q$, we have

$$
\sum_{\substack{\mathcal{N} \mathfrak{p} \in[X, 2 X] \\ \mathfrak{p} \text { unramified }}} \sum_{\substack{1 \leq i \leq r\\}}\left|\alpha_{i, \rho}(\mathfrak{p})-\alpha_{i, \rho^{\prime}}(\mathfrak{p})\right| \geq \frac{X}{2 r \log X} .
$$

Furthermore, the number of representations of degree $\leq r$ and conductor $\leq q$ is at most

$$
C_{17}^{r^{3} \log ^{2}(q)[K: \mathbb{Q}]}
$$

for an absolute constant $C_{17}$.

Remark. The bound (4-2) is rather simple-minded, and we could remove the factor $[K: \mathbb{Q}]$ by instead insisting that $q$ be sufficiently large in terms of $K$.

Proof. This is essentially Proposition 5.22 of [Iwaniec and Kowalski 2004]; although our conclusion is stronger, our proof is essentially the same.

Consider the tensor product representations $\rho \otimes \bar{\rho}$ and $\rho \otimes \overline{\rho^{\prime}}$, whose $L$-functions are equal to the Rankin-Selberg convolutions $L(s, \rho \otimes \bar{\rho})$ and $L\left(s, \rho \otimes \overline{\rho^{\prime}}\right)$ (so that the notation is not ambiguous). A simple character-theoretic argument shows that the trivial representation does not occur in $\rho \otimes \overline{\rho^{\prime}}$, while it occurs with multiplicity one in $\rho \otimes \bar{\rho}$. Assuming the Artin conjecture, then, $L\left(s, \rho \otimes \overline{\rho^{\prime}}\right)$ and $L(s, \rho \otimes \bar{\rho}) \zeta(s)^{-1}$ are entire functions.

Let $\phi$ be a smooth test function with support in [1,2], image in [0,1], and $\widehat{\phi}(0)=\int_{1}^{2} \phi(t) d t \in\left(\frac{3}{4}, 1\right)$; throughout this section, all implied constants (including 
$C_{16}$, etc.) depend on our fixed choice of $\phi$. Also, let $X \geq 2$, with stricter lower bounds to be imposed later. Then, using the "explicit formula" [ibid., Theorem 5.11] we find (see p. 118 of [ibid.]) that, assuming GRH and the Artin conjecture,

$$
\begin{gathered}
\left|\sum_{n} \Lambda_{\rho \otimes \bar{\rho}^{\prime}}(n) \phi(n / X)\right| \ll \sqrt{X} \log \mathfrak{q}\left(\rho \otimes \overline{\rho^{\prime}}\right), \\
\left|\sum_{n} \Lambda_{\rho \otimes \bar{\rho}}(n) \phi(n / X)-\widehat{\phi}(0) X\right| \ll \sqrt{X} \log \mathfrak{q}(\rho \otimes \bar{\rho}),
\end{gathered}
$$

where the coefficients $\Lambda$ are defined, for any $L$-function $L(f, s)$, by the relation

$$
\sum_{n} \Lambda_{f}(n) n^{-s}=-\frac{L^{\prime}}{L}(f, s) .
$$

Also, $\mathfrak{q}(\rho)=q(\rho) \prod_{j=1}^{r}\left(\left|\kappa_{j}\right|+3\right)$ denotes the analytic conductor of $\rho$ defined by equation (5.8) of [ibid.]. To bound this analytic conductor, we require information about the gamma factors of Artin $L$-functions and the conductor of $\rho \otimes \psi$ (where $\psi=\bar{\rho}$ or $\left.\overline{\rho^{\prime}}\right)$.

The fact that the $L(s, \rho)$ are factors of the Dedekind zeta function and the fact that the Dedekind zeta function only has gamma factors $\Gamma(s / 2)$ and $\Gamma((s+1) / 2)$ imply that $0 \leq \kappa_{j} \leq 1 / 2$ for all $j$. We have $q(\rho \otimes \psi)=|\operatorname{Disc}(K)|^{\operatorname{deg}(\rho \otimes \psi)} \mathcal{N}_{K / \mathbb{Q}}(\mathfrak{f}(\rho \otimes \psi))$, where

$$
\mathfrak{f}(\rho \otimes \psi)=\prod_{\mathfrak{p} \nmid \infty} \mathfrak{p}^{\mathfrak{f}_{\mathfrak{p}}(\rho \otimes \psi)}, \quad f_{\mathfrak{p}}(\rho \otimes \psi)=\sum_{i=0}^{\infty} \frac{g_{i}}{g_{0}} \operatorname{codim} V_{\rho \otimes \psi}^{G_{i}} .
$$

Here $V_{\rho \otimes \psi}$ is a vector space affording the representation $\rho \otimes \psi, G_{i}$ is the $i$-th ramification group, and $g_{i}=\left|G_{i}\right|$ (this definition is from p. 527 of [Neukirch 1999]). It is easy to see that $\operatorname{codim} V_{\rho \otimes \psi}^{G_{i}} \leq r \operatorname{codim} V_{\rho}^{G_{i}}+r \operatorname{codim} V_{\psi}^{G_{i}}$. It follows from this and the formulas

$$
f_{\mathfrak{p}}(\rho)=\sum_{i=0}^{\infty} \frac{g_{i}}{g_{0}} \operatorname{codim} V_{\rho}^{G_{i}} \quad \text { and } \quad f_{\mathfrak{p}}(\psi)=\sum_{i=0}^{\infty} \frac{g_{i}}{g_{0}} \operatorname{codim} V_{\psi}^{G_{i}} .
$$

that $f_{\mathfrak{p}}(\rho \otimes \psi) \leq r\left(f_{\mathfrak{p}}(\rho)+f_{\mathfrak{p}}(\psi)\right)$ and

$$
q(\rho \otimes \psi) \leq|\operatorname{Disc}(K)|^{r^{2}} \mathcal{N}_{K / \mathbb{Q}}(\mathfrak{f}(\rho) \mathfrak{f}(\psi)) .
$$

Combining these estimates for the analytic conductor yields the bound

$$
\log \mathfrak{q}(\rho \otimes \psi) \leq\left(2 r^{2}+r^{2} \log |\operatorname{Disc}(K)|+r \log \mathcal{N}_{K / \mathbb{Q}}(\mathfrak{f}(\rho) \mathfrak{f}(\psi))\right) \leq 3 r \log q .
$$

Let $\alpha_{i, \rho}$ and $\alpha_{i, \rho^{\prime}}$ be the Frobenius eigenvalues for $\rho$ and $\rho^{\prime}$ respectively, for $1 \leq i \leq r$. Then, unpacking the definition of the Rankin-Selberg convolution (or, equivalently, 
the tensor product representation), we conclude that

$$
\begin{aligned}
\sum_{\mathcal{N} \mathfrak{p} \in[X, 2 X]} \sum_{1 \leq i, j \leq r}\left|\alpha_{i, \rho}(\mathfrak{p}) \overline{\alpha_{j, \rho}(\mathfrak{p})}-\alpha_{i, \rho}(\mathfrak{p}) \overline{\alpha_{j, \rho^{\prime}}(\mathfrak{p})}\right| & \\
& \geq \frac{\widehat{\phi}(0) X}{\log (2 X)}-\frac{C_{18} \sqrt{X}}{\log X}(r \log q) .
\end{aligned}
$$

The sum above is over prime ideals; we have removed the prime powers which contribute (assuming $X$ is large) less than

$$
\frac{2 r^{2}[K: \mathbb{Q}] \sqrt{X}}{\log X}<\frac{2 r \log (q) \sqrt{X}}{\log X},
$$

which is contained in the error term above. Next, we remove the terms coming from "ramified primes", those for which $\alpha_{i, \rho}(\mathfrak{p})=0$ for some $i$. These occur precisely at the primes for which $\mathfrak{p} \mid \mathfrak{f}(\rho)$, and the number of these between $X$ and $2 X$ is at most $\log q / \log X$. Noting that for a prime $\mathfrak{p}$ that is unramified in $L / K,\left|\alpha_{i, \rho}(\mathfrak{p})\right|=1$ for all $i$, we get

$$
\sum_{\substack{\mathcal{N} \in[X, 2 X] \\ \mathfrak{p} \text { unramified }}} \sum_{1 \leq j \leq r}\left|\alpha_{j, \rho}(\mathfrak{p})-\alpha_{j, \rho^{\prime}}(\mathfrak{p})\right| \geq \frac{\widehat{\phi}(0) X}{r \log (2 X)}-C_{19}\left(\frac{\sqrt{X} \log q+r \log q}{\log X}\right)
$$

If $X \geq C_{16} r^{2} \log ^{2}(q)$ with $C_{16}=\max \left(2^{14}, 100 C_{19}^{2}\right)$, the error term above is at most $X /(5 r \log X)$ and the main term is at least $7 X /(10 r \log X)$, establishing the first part of the proposition.

The second part follows easily from the first. Let $M$ be the number of primes in the above sum; then, if

$$
\left|\alpha_{i, \rho}(\mathfrak{p})-\alpha_{i, \rho^{\prime}}(\mathfrak{p})\right| \leq \frac{X}{2 r^{2} \log (X) M}
$$

for all $i$ and unramified $\mathfrak{p}$, then (4-1) is contradicted. Note that since $\mathfrak{p}$ is unramified, $\alpha_{i, \rho}(\mathfrak{p})$ and $\alpha_{i, \rho^{\prime}}(\mathfrak{p})$ lie on the unit circle. No more than $2 \pi Y$ points can be placed on the unit circle with pairwise distances at least $1 / Y$. Hence, by the pigeonhole principle, there can be at most

$$
N=\left(\frac{4 \pi r^{2} \log (X) M}{X}\right)^{r\left(\pi_{K}(2 X)-\pi_{K}(X)\right)}
$$

Artin $L$-functions of degree $r$ and conductor $\leq q$. Here, $\pi_{K}(X)$ is the number of prime ideals of $0_{K}$ of norm at most $X$. We have $M \leq \pi_{K}(2 X)-\pi_{K}(X) \leq$ $2[K: \mathbb{Q}] X / \log X$ and so

$$
\log N \leq \frac{2 r X[K: \mathbb{Q}]}{\log X}\left(\log \left(8 \pi r^{2}\right)+\log [K: \mathbb{Q}]\right)
$$


We have

$$
\log X \geq \log 2^{14}+2 \log r+2 \log \log q>\log 8 \pi+2 \log r+\log [K: \mathbb{Q}],
$$

and hence

$$
\log N \leq 2 C_{16} r^{3} \log ^{2}(q)[K: \mathbb{Q}] .
$$

As $e^{r^{3} \log ^{2}(q)[K: \mathbb{Q}]}$ increases rapidly in $r$, the number of representations of conductor at most $q$ and degree $r^{\prime} \leq r$ is bounded by $C_{17}^{r^{3} \log ^{2}(q)[K: \mathbb{Q}]}$, for some absolute constant $C_{17}$.

We now prove Theorem 1.2 using Proposition 4.1.

Proof of Theorem 1.2. Assume $L / K$ is a Galois extension whose irreducible complex representations all have degree at most $r$. Choose the smallest $A \geq e$ such that

$$
\frac{[L: K]}{2 r^{2}} \leq \sum_{i=1}^{r} C_{17}^{r^{2}(r-i) \log ^{2}\left(A^{2}\right)[K: \mathbb{Q}]} .
$$

We wish to estimate $A$ in terms of $|\operatorname{Disc}(L)|$. By Proposition 4.1, the number of representations with degree $r-i$ and conductor $\leq A^{2 r /(r-i)}$ is at most

$$
C_{17}^{(r-i)^{3} \log ^{2}\left(A^{2 r /(r-i)}\right)[K: \mathbb{Q}]}=C_{17}^{4 r^{2}(r-i) \log ^{2}\left(A^{2}\right)[K: \mathbb{Q}]} .
$$

Every other representation has $q(\rho)^{\operatorname{deg} \rho} \geq A^{2 r}$. There are at least $[L: K] / 2 r^{2}$ of these, and so (2-8) gives

$$
|\operatorname{Disc}(L)|=\prod_{\rho \in \operatorname{Irr}(\operatorname{Gal}(L / K))} q(\rho)^{\operatorname{deg} \rho} \geq\left(A^{2 r}\right)^{[L: K] / 2 r^{2}}=A^{[L: K] / r} .
$$

Thus, $\log A \leq(r /[L: K]) \log |\operatorname{Disc}(L)|=r \cdot[K: \mathbb{Q}] \log \operatorname{rd}_{L}$.

Equation (4-8) gives

$$
\frac{[L: K]}{2 r^{2}} \leq 2 C_{17}^{r^{3} \log ^{2}\left(A^{2}\right)[K: \mathbb{Q}]}
$$

enlarging $C_{17}$ if necessary so that $C_{17}^{e^{2}}>2$, which gives

$$
\begin{aligned}
\log [L: K] & \leq \log \left(4 r^{2}\right)+r^{3} \log ^{2}(A)[K: \mathbb{Q}] \log C_{17} \\
& \leq\left(\log 4+\log C_{17}\right) \cdot r^{5}[K: \mathbb{Q}]^{3}\left(\log \operatorname{rd}_{L}\right)^{2} .
\end{aligned}
$$

Hence, there is an absolute constant $C_{2}$ so that

$$
r \geq C_{2} \frac{(\log [L: K])^{1 / 5}}{[K: \mathbb{Q}]^{3 / 5}\left(\log \operatorname{rd}_{L}\right)^{2 / 5}} .
$$

Finally, we prove Corollary 1.3 using Proposition 4.1. 
Proof of Corollary 1.3. Let $L$ be the compositum of the $L_{i}$. Then we have

$$
\operatorname{Gal}(L / K)=\prod_{i=1}^{N} \operatorname{Gal}\left(L_{i} / K\right) .
$$

From Theorem 4.21 of [Isaacs 2006], if $\rho_{i}: \operatorname{Gal}\left(L_{i} / K\right) \rightarrow \mathrm{GL}_{r}(\mathbb{C})$ is an irreducible representation, then the map $\tilde{\rho}_{i}(g)=\rho_{i}\left(\left.g\right|_{L_{i}}\right)$ is also an irreducible representation of $\operatorname{Gal}(L / K)$ which is distinct from $\tilde{\rho}_{j}$ for $i \neq j$. All of these representations have conductor $q=|\operatorname{Disc}(K)|^{r}$, and Proposition 4.1 implies that there is an absolute constant $C_{3}$ so that

$$
\log N \leq C_{3} r^{5} \log ^{2}(|\operatorname{Disc}(K)|)[K: \mathbb{Q}]
$$

as desired.

\section{Acknowledgments}

We would like to thank Kannan Soundararajan for suggesting the basic idea of this paper to us. We'd also like to thank Charlotte Euvrard and the referee for a number of very helpful suggestions.

\section{References}

[Cojocaru and Murty 2006] A. C. Cojocaru and M. R. Murty, An introduction to sieve methods and their applications, London Mathematical Society Student Texts 66, Cambridge University Press, 2006. MR 2006k:11184 Zbl 1121.11063

[Ellenberg and Venkatesh 2006] J. S. Ellenberg and A. Venkatesh, "The number of extensions of a number field with fixed degree and bounded discriminant”, Ann. of Math. (2) 163:2 (2006), 723-741. MR 2006j:11159 Zbl 1099.11068

[Golod and Shafarevich 1964] E. S. Golod and I. R. Shafarevich, "О башне полей классов”, Izv. Akad. Nauk SSSR Ser. Mat. 28:2 (1964), 261-272. Translated as "On the class field tower" in Amer. Math. Soc. Transl. 48 (1965), 91-102. MR 28 \#5056 Zbl 0136.02602

[Hajir and Maire 2001] F. Hajir and C. Maire, "Tamely ramified towers and discriminant bounds for number fields", Compositio Math. 128:1 (2001), 35-53. MR 2002g:11149 Zbl 1042.11072

[Hajir and Maire 2002] F. Hajir and C. Maire, "Tamely ramified towers and discriminant bounds for number fields, II”, J. Symbolic Comput. 33:4 (2002), 415-423. MR 2003h:11137 Zbl 1086.11051

[Isaacs 2006] I. M. Isaacs, Character theory of finite groups, AMS Chelsea, Providence, RI, 2006. MR 2270898 Zbl 1119.20005

[Iwaniec and Kowalski 2004] H. Iwaniec and E. Kowalski, Analytic number theory, American Mathematical Society Colloquium Publications 53, American Mathematical Society, Providence, RI, 2004. MR 2005h:11005 Zbl 1059.11001

[Jones and Roberts 2007] J. W. Jones and D. P. Roberts, "Galois number fields with small root discriminant”, J. Number Theory 122:2 (2007), 379-407. MR 2008e:11140 Zbl 1163.11079 
[Lagarias and Odlyzko 1977] J. C. Lagarias and A. M. Odlyzko, "Effective versions of the Chebotarev density theorem", pp. 409-464 in Algebraic number fields: L-functions and Galois properties (Durham, 1975), edited by A. Fröhlich, Academic Press, London, 1977. MR 56 \#5506 Zbl 0362.12011

[Leshin 2013] J. Leshin, "Solvable number field extensions of bounded root discriminant", Proc. Amer. Math. Soc. 141:10 (2013), 3341-3352. MR 3080157 Zbl 06203295

[Martinet 1979] J. Martinet, "Petits discriminants", Ann. Inst. Fourier (Grenoble) 29:1 (1979), 159170. MR 81h:12006 Zbl 0387.12006

[Michel and Venkatesh 2002] P. Michel and A. Venkatesh, "On the dimension of the space of cusp forms associated to 2-dimensional complex Galois representations", Int. Math. Res. Not. 2002:38 (2002), 2021-2027. MR 2003i:11064 Zbl 1008.11019

[Milne 2011] J. S. Milne, "Class field theory", course notes, v. 4.01, 2011, http://www.jmilne.org/ math/CourseNotes/cft.html.

[Neukirch 1999] J. Neukirch, Algebraic number theory, Grundlehren der Mathematischen Wissenschaften 322, Springer, Berlin, 1999. MR 2000m:11104 Zbl 0956.11021

[Odlyzko 1976] A. M. Odlyzko, "Lower bounds for discriminants of number fields", Acta Arith. 29:3 (1976), 275-297. MR 53 \#5531 Zbl 0286.12006

[Odlyzko 1990] A. M. Odlyzko, "Bounds for discriminants and related estimates for class numbers, regulators and zeros of zeta functions: a survey of recent results", Sém. Théor. Nombres Bordeaux (2) 2:1 (1990), 119-141. MR 91i:11154 Zbl 0722.11054

[Poitou 1977] G. Poitou, "Minorations de discriminants (d'après A. M. Odlyzko)", pp. 136-153 (Exp. No. 479) in Séminaire Bourbaki, Vol. 1975/76, Lecture Notes in Math. 567, Springer, Berlin, 1977. MR 55 \#7995 Zbl 0359.12010

[Stark 1974] H. M. Stark, "Some effective cases of the Brauer-Siegel theorem", Invent. Math. 23 (1974), 135-152. MR 49 \#7218 Zbl 0278.12005

[Voight 2008] J. Voight, "Enumeration of totally real number fields of bounded root discriminant", pp. 268-281 in Algorithmic number theory (Banff, 2008), edited by A. J. van der Poorten and A. Stein, Lecture Notes in Comput. Sci. 5011, Springer, Berlin, 2008. MR 2010a:11228 Zbl 1205.11125

Received March 4, 2013. Revised June 17, 2014.

JEREMY ROUSE

DEPARTMENT OF MATHEMATICS

WAKE FOREST UNIVERSITY

P.O. BOX 7388

WINSTON-SALEM, NC 27109

UNITED STATES

rouseja@wfu.edu

FRANK THORNE

DePartment of MATHEMATiCs

UNIVERSITY OF SOUTH CAROLINA

1523 GREene STREet

COLUMbia, SC 29208

UNITED STATES

thorne@math.sc.edu 


\title{
PACIFIC JOURNAL OF MATHEMATICS
}

\author{
msp.org/pjm
}

Founded in 1951 by E. F. Beckenbach (1906-1982) and F. Wolf (1904-1989)

\section{EDITORS}

Don Blasius (Managing Editor)

Department of Mathematics

University of California

Los Angeles, CA 90095-1555

blasius@math.ucla.edu

\author{
Paul Balmer \\ Department of Mathematics \\ University of California \\ Los Angeles, CA 90095-1555 \\ balmer@math.ucla.edu \\ Robert Finn \\ Department of Mathematics \\ Stanford University \\ Stanford, CA 94305-2125 \\ finn@math.stanford.edu \\ Sorin Popa \\ Department of Mathematics \\ University of California \\ Los Angeles, CA 90095-1555 \\ popa@math.ucla.edu
}

\author{
Vyjayanthi Chari \\ Department of Mathematics \\ University of California \\ Riverside, CA 92521-0135 \\ chari@math.ucr.edu \\ Kefeng Liu \\ Department of Mathematics \\ University of California \\ Los Angeles, CA 90095-1555 \\ liu@math.ucla.edu \\ Jie Qing \\ Department of Mathematics \\ University of California \\ Santa Cruz, CA 95064 \\ qing@ cats.ucsc.edu
}

\section{PRODUCTION}

Silvio Levy, Scientific Editor, production@msp.org

\section{SUPPORTING INSTITUTIONS}

ACADEMIA SINICA, TAIPEI

CALIFORNIA INST. OF TECHNOLOGY

INST. DE MATEMÁTICA PURA E APLICADA

KEIO UNIVERSITY

MATH. SCIENCES RESEARCH INSTITUTE

NEW MEXICO STATE UNIV.

OREGON STATE UNIV.

\author{
STANFORD UNIVERSITY \\ UNIV. OF BRITISH COLUMBIA \\ UNIV. OF CALIFORNIA, BERKELEY \\ UNIV. OF CALIFORNIA, DAVIS \\ UNIV. OF CALIFORNIA, LOS ANGELES \\ UNIV. OF CALIFORNIA, RIVERSIDE \\ UNIV. OF CALIFORNIA, SAN DIEGO \\ UNIV. OF CALIF., SANTA BARBARA
}

\author{
Daryl Cooper \\ Department of Mathematics \\ University of California \\ Santa Barbara, CA 93106-3080 \\ cooper@math.ucsb.edu \\ Jiang-Hua Lu \\ Department of Mathematics \\ The University of Hong Kong \\ Pokfulam Rd., Hong Kong \\ jhlu@maths.hku.hk \\ Paul Yang \\ Department of Mathematics \\ Princeton University \\ Princeton NJ 08544-1000 \\ yang@math.princeton.edu
}

These supporting institutions contribute to the cost of publication of this Journal, but they are not owners or publishers and have no responsibility for its contents or policies.

See inside back cover or msp.org/pjm for submission instructions.

The subscription price for 2014 is US $\$ 410 /$ year for the electronic version, and \$535/year for print and electronic.

Subscriptions, requests for back issues and changes of subscribers address should be sent to Pacific Journal of Mathematics, P.O. Box 4163, Berkeley, CA 94704-0163, U.S.A. The Pacific Journal of Mathematics is indexed by Mathematical Reviews, Zentralblatt MATH, PASCAL CNRS Index, Referativnyi Zhurnal, Current Mathematical Publications and Web of Knowledge (Science Citation Index).

The Pacific Journal of Mathematics (ISSN 0030-8730) at the University of California, c/o Department of Mathematics, 798 Evans Hall \#3840, Berkeley, CA 94720-3840, is published twelve times a year. Periodical rate postage paid at Berkeley, CA 94704, and additional mailing offices. POSTMASTER: send address changes to Pacific Journal of Mathematics, P.O. Box 4163, Berkeley, CA 94704-0163.

PJM peer review and production are managed by EditFLOW ${ }^{\circledR}$ from Mathematical Sciences Publishers.

\section{PUBLISHED BY}

\section{mathematical sciences publishers \\ nonprofit scientific publishing}

http://msp.org/

(C) 2014 Mathematical Sciences Publishers 


\section{PACIFIC JOURNAL OF MATHEMATICS}

Volume $271 \quad$ No. $1 \quad$ September 2014

Proper holomorphic maps between bounded symmetric domains revisited 1

GAUTAM BHARALI and JAIKRISHNAN JANARDHANAN

An explicit Majorana representation of the group $3^{2}: 2$ of $3 C$-pure type 25

HSIAN-YANG CHEN and CHING HUNG LAM

Sofic groups: graph products and graphs of groups

LAUra Ciobanu, DereK F. Holt and SARAh ReES

Perturbations of a critical fractional equation

Eduardo Colorado, Arturo de Pablo and Urko SÁnchez

A density theorem in parametrized differential Galois theory

THOMAS DREYFUS

On the classification of complete area-stationary and stable surfaces in $\quad 143$ the subriemannian Sol manifold

MATteo Galli

Periodic orbits of Hamiltonian systems linear and hyperbolic at infinity

BAŞAK Z. GÜREL

Nonsplittability of the rational homology cobordism group of

3-manifolds

SE-Goo KIM and CHARLES LIVINGSTON

Biharmonic surfaces of constant mean curvature

ERIC LOUBEAU and CEZAR ONICIUC

Foliations of a smooth metric measure space by hypersurfaces with constant $f$-mean curvature

JUNCHEOL PYO

On the existence of large degree Galois representations for fields of small 243 discriminant

JEREMY ROUSE and FRANK THORNE 